Wirtschaftswissenschaftliches Zentrum (WWZ) der Universität Basel

$\mathrm{WW} \mid \mathrm{Z}$

December 2014

\title{
Existence of Steady-State Equilibria in Matching Models with Search Frictions
}


Corresponding author:

\section{Prof. Georg Nöldeke}

University of Basel

Faculty of Business and Economics

Peter Merian-Weg 6

CH-4002 Basel

Phone: +41(0)612673320

georg.noeldeke@unibas.ch

A publication of the Center of Business and Economics (WWZ), University of Basel.

(c) WWZ 2014 and the authors. Reproduction for other purposes than the personal use needs the permission of the authors.

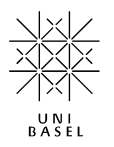




\title{
Existence of Steady-State Equilibria in Matching Models with Search Frictions
}

\author{
Stephan Lauermann ${ }^{\mathrm{a}}$, Georg Nöldeke ${ }^{\mathrm{b}}$ \\ ${ }^{a}$ Department of Economics, University of Bonn, Germany \\ ${ }^{b}$ Faculty of Business and Economics, University of Basel, Switzerland
}

\begin{abstract}
We prove existence of steady-state equilibrium in a class of matching models with search frictions.

JEL classification: C62, C78, D83.
\end{abstract}

Keywords: Search, Matching, Bargaining, Steady-State Equilibrium

\section{Introduction}

This paper contributes to the literature on the pairwise matching of heterogenous agents with search frictions. The basic structure of our model is as in Shimer and Smith (2000) or Smith (2006): There is a continuum of infinitely lived agents who are either matched or unmatched at any moment in time. Meetings between unmatched agents are generated by an exogenous search technology. Upon meeting, agents play a bargaining game, determining whether or not they become matched and provided they do so, their payoffs within the match. The main concern of the literature studying such models (surveyed in Smith, 2011) is the characterization of matching patterns in steady-state equilibria. Here we focus on the question of existence of steadystate equilibria.

Previously, this question has been addressed using two distinct approaches. Shimer and Smith (2000) provide an existence argument applicable both to models with transferable and nontransferable utility (see Smith, 2006),

Email addresses: s.lauermann@uni-bonn.de (Stephan Lauermann), georg.noeldeke@unibas.ch (Georg Nöldeke) 
but requiring that - given the agents' decisions which matches to acceptthere is a unique distribution of unmatched agents which maintains a steady state. To obtain this uniqueness property, Shimer and Smith (2000) impose the stringent assumption of a quadratic search technology. ${ }^{1}$ In contrast, the approach developed in Manea (2014a) accommodates general search technologies, but requires the uniqueness of equilibrium payoffs in an auxiliary model in which the distribution of unmatched agents searching for a partner is taken as given. While Manea (2014a) establishes this uniqueness property for a model with transferable utility, it is evident from Adachi (2003) that, in general, this property fails with nontransferable utility.

In the following we develop an approach to proving existence of steadystate equilibrium which dispenses with the uniqueness requirements in Shimer and Smith (2000) and Manea (2014a). This yields existence under minimal regularity conditions on the search technology, akin to the ones introduced in Manea (2014a), and the bargaining problem faced by the agents when deciding on a match, allowing for both transferable and nontransferable utility.

\section{Model}

There is an exogenous measure $\theta_{i}>0$ of players of type $i \in N=\{1, \ldots, n\}$, or simply players $i .^{2}$ Players are risk neutral, infinitely lived, and discount payoffs at rate $r>0$. Time is continuous. We consider steady states in which a measure $\mu_{i}>0$ of players $i$ is unmatched and a measure $\theta_{i}-\mu_{i}>0$ is matched. The unmatched players search: each unmatched player $i$ meets unmatched players $j$ at Poisson rate $\rho_{i j}(\mu) \geq 0$, where $\mu=\left(\mu_{1}, \ldots, \mu_{n}\right) \in \mathbb{R}_{++}^{n}$.

Assumption 1. There exists a continuous function $m: \mathbb{R}_{+}^{n} \rightarrow \mathbb{R}_{+}^{n \times n}$, satisfying (i) $m_{i j}(\mu)=m_{j i}(\mu)$ for all $\mu \in \mathbb{R}_{+}^{n}$ and (ii) $m_{i j}(\mu)=0$ whenever $\mu_{i}=0$, such that

$$
\rho_{i j}(\mu)=\frac{m_{i j}(\mu)}{\mu_{i}}, \quad \forall \mu \in \mathbb{R}_{++}^{n} .
$$

\footnotetext{
${ }^{1}$ While Nöldeke and Tröger (2009) refine the existence argument from Shimer and Smith (2000) to cover linear search technologies neither proof extends to general search technologies. See Manea (2014a) for further discussion.

${ }^{2}$ We follow Manea (2014a) in considering a finite type space rather than a continuum of types, thus sidestepping technicalities - but not, as discussed in Smith (2011), the substantive issues - in Shimer and Smith (2000). In a similar vein, we follow most of the literature in suppressing the measure theoretic considerations discussed and resolved in Manea (2014b).
} 
Assumption 1 is similar to the assumption on meeting rates in Manea (2014a). From equation $(1), m_{i j}(\mu)=\rho_{i j}(\mu) \mu_{i}$. Hence, $m_{i j}(\mu)$ is the mass of players $i$ who meet a player $j$ per unit time, which - as stated in part (i) of Assumption 1 - should equal the mass $m_{j i}(\mu)=\rho_{j i}(\mu) \mu_{j}$ of players $j$ who meet a player $i$ per unit time. As the mass of meetings is assumed continuous in the distribution of unmatched types, part (ii) of Assumption 1 is the natural boundary condition that the mass of meetings involving players $i$ approaches zero as $\mu_{i}$ vanishes (see Stevens, 2007).

When unmatched players $i$ and $j$ meet, they play a bargaining game determining whether or not they form a match - enter a relationship - and if they do, the flow payoffs that they obtain until their match dissolves. Each match is dissolved randomly at Poisson rate $\omega>0$. Separated partners return to the pool of unmatched agents. If players do not agree to match, they instantaneously return to the pool of unmatched agents.

If a fraction $a_{i j} \in[0,1]$ of meetings between unmatched agents $i$ and $j$ results in a match, then the outflow of players $i$ from the pool of unmatched agents per unit time is $\sum_{j \in N} a_{i j} m_{i j}(\mu)$. The inflow of such players into the unmatched pool is given by the mass of matched players $i$ multiplied with the exogenous rate at which the matches of such players are dissolved, i.e., $\omega\left(\theta_{i}-\mu_{i}\right)$. In a steady state, inflows and outflows must balance, delivering the balance condition

$$
\omega\left(\theta_{i}-\mu_{i}\right)=\sum_{j \in N} a_{i j} m_{i j}(\mu), \quad \forall i \in N
$$

Denote by $v_{i}$ the expected (continuation) value of an unmatched player $i$ and by $v_{i j}$ the expected value of a player $i$ conditional on a meeting with a player $j$ before the bargaining in the pair has commenced. The flow payoff of unmatched players is zero. Because a player $i$ meets a player $j$ at rate $\rho_{i j}(\mu)$ and such a meeting results in a capital gain of $g_{i j}=v_{i j}-v_{i}$, we have the value condition

$$
r v_{i}=\sum_{j \in N} \rho_{i j}(\mu) g_{i j}, \quad \forall i \in N .
$$

Agents are free to refuse to enter a relationship, so that $v_{i} \geq 0$ and $g_{i j} \geq 0$. In the following $v=\left(v_{1}, \ldots, v_{n}\right) \in \mathbb{R}_{+}^{n}$ denotes the vector of continuation values, the matrix $g \in \mathbb{R}_{+}^{n \times n}$ collects the gains $g_{i j}$, and the set of feasible matching probabilities $a_{i j}$ is

$$
A=\left\{a \in[0,1]^{n \times n} \mid a_{i j}=a_{j i}, \forall(i, j) \in N \times N\right\} .
$$


The matching probabilities $a_{i j}$ and the gains $g_{i j}$ are determined by an equilibrium in the bargaining game between players $i$ and $j$. We treat the bargaining game as a "black box" by specifying a bargaining correspondence $E: \mathbb{R}_{+}^{n} \rightrightarrows A \times \mathbb{R}_{+}^{n \times n}$, mapping vectors of continuation values $v$ into outcomes $(a, g)$. The interpretation is that $(a, g)$ is an equilibrium outcome in the collection of bilateral bargaining games induced by a vector of continuation values $v$ if and only if $(a, g)$ satisfies the bargaining condition

$$
(a, g) \in E(v) .
$$

Assumption 2. The bargaining correspondence $E: \mathbb{R}_{+}^{n} \rightrightarrows A \times \mathbb{R}_{+}^{n \times n}$ is upper hemicontinuous with $E(v)$ non-empty, closed, and convex-valued for all $v \in \mathbb{R}_{+}^{n}$. Further, there exists $\bar{g} \in \mathbb{R}_{+}$such that $E(v) \subset A \times[0, \bar{g}]^{n \times n}$ holds for all $v \in \mathbb{R}_{+}^{n}$.

Section 4 derives the bargaining correspondence for two common specifications of the bargaining problem. In both cases, Assumption 2 is satisfied.

Definition 1. A steady-state equilibrium is a tuple $(\mu, v, a, g) \in \mathbb{R}_{++}^{n} \times \mathbb{R}_{+}^{n} \times$ $A \times \mathbb{R}_{+}^{n \times n}$ satisfying the balance condition (2), the value condition (3), and the bargaining condition (5).

\section{Result}

Proposition 1. A steady-state equilibrium exists if Assumptions 1 and 2 hold.

The idea underlying the proof of Proposition 1 is as follows: We may rewrite the balance condition (2) as

$$
\mu_{i}=\frac{\theta_{i}-\sum_{j \in N} a_{i j} m_{i j}(\mu)}{\omega}, \forall i \in N
$$

and the value condition (3) as

$$
v_{i}=\frac{\sum_{j \in N} \rho_{i j}(\mu) g_{i j}}{r}, \quad \forall i \in N .
$$

Together with the bargaining condition (5) the right sides of (6) and (7) define a mapping $(\mu, v, a, g) \longmapsto\left(\mu^{\prime}, v^{\prime}, a^{\prime}, g^{\prime}\right)$. Fixed points of this mapping 
coincide with steady-state equilibria. Assumptions 1 and 2 then ensure that the existence of steady-state equilibria can be inferred from Kakutani's fixed point theorem.

The key difference to the fixed point argument in Shimer and Smith (2000) is that these authors, rather than using the equilibrium conditions in a parallel fashion as we do, compose them to define a mapping $v \longmapsto v^{\prime}$. For this mapping to be well-behaved, it is required that the balance condition has a unique solution $\mu$ for any given $a$. Similarly, Manea (2014a) composes the equilibrium conditions to define a mapping $\mu \longmapsto \mu^{\prime}$ and requires that, for given $\mu$, the equilibrium conditions determine $(v, g)$ uniquely for this mapping to be well-behaved.

Proof of Proposition 1. Let $\tilde{M}=\prod_{i \in N}\left[0, \theta_{i}\right]$. Define the function $\zeta: \tilde{M} \rightarrow$ $\mathbb{R}^{n}$ by

$$
\zeta_{i}(\mu)=\omega\left(\mu_{i}-\theta_{i}\right)+\sum_{j \in N} m_{i j}(\mu), \quad \forall i \in N .
$$

Using Assumption 1, $\zeta_{i}(\mu)=-\omega \theta_{i}<0$ holds whenever $\mu_{i}=0$. Further, $\zeta$ is uniformly continuous on $\tilde{M}$ because $\tilde{M}$ is compact. Hence, there exists $\eta_{i} \in\left(0, \theta_{i}\right)$ such that $\zeta_{i}(\mu)<0$ holds whenever $\mu_{i} \leq \eta_{i}$. Pick such $\eta_{i}>0$ for all $i \in N$ and let

$$
M=\prod_{i \in N}\left[\eta_{i}, \theta_{i}\right]
$$

The set $M$ is non-empty, compact, and convex. By Assumption 1, the meeting rates $\rho_{i j}$ are continuous on the compact set $M$ and, thus, bounded above by some $\bar{\rho}>0$ on $M$. Define

$$
V=\prod_{i \in N}\left[0, \frac{n \bar{\rho} \bar{g}}{r}\right]
$$

which is non-empty, compact, and convex.

Define the function $\psi^{\mu}: M \times A \rightarrow M$ by

$$
\psi_{i}^{\mu}(\mu, a)=\max \left\{\theta_{i}-\frac{\sum_{j \in N} a_{i j} m_{i j}(\mu)}{\omega}, \eta_{i}\right\}, \quad \forall i \in N .
$$

By continuity of $m_{i j}$ (Assumption 1), the function $\psi^{\mu}$ is continuous. The set $A$, defined in (4), is compact, convex, and non-empty. 
From Assumption 2 there exists $\bar{g} \in \mathbb{R}_{++}$such that $E(v) \subset A \times[0, \bar{g}]^{n \times n}$ holds for all $v \in \mathbb{R}_{+}^{n}$. Let $G=[0, \bar{g}]^{n \times n}$, which is non-empty, compact, and convex. Define the continuous function $\psi^{v}: M \times G \rightarrow V$ by

$$
\psi_{i}^{v}(\mu, g)=\frac{\sum_{j \in N} \rho_{i j}(\mu) g_{i j}}{r}, \forall i \in N .
$$

Observing that the restriction of the bargaining correspondence $E$ to the domain $V$ satisfies Assumption 2, the correspondence $\psi: M \times V \times A \times G \rightrightarrows$ $M \times V \times A \times G$, defined by

$$
\psi(\mu, v, a, g)=\left(\left\{\psi^{\mu}(\mu, a)\right\},\left\{\psi^{v}(\mu, g)\right\}, E(v)\right),
$$

then satisfies the assumptions of Kakutani's fixed point theorem. Hence, there exists $\left(\mu^{*}, v^{*}, a^{*}, g^{*}\right) \in M \times V \times A \times G$ such that $\left(\mu^{*}, v^{*}, a^{*}, g^{*}\right)=$ $\psi\left(\mu^{*}, v^{*}, a^{*}, g^{*}\right)$.

It remains to show that such a fixed point of $\psi$ satisfies conditions (2), (3), and (5). For conditions (3) and (5) this is immediate from the construction of $\psi$. It remains to establish (2). This follows from (11), provided that

$$
\theta_{i}-\frac{\sum_{j \in N} a_{j i}^{*} m_{i j}\left(\mu^{*}\right)}{\omega} \geq \eta_{i}, \quad \forall i \in N .
$$

Suppose not. From (11) there then exists $i \in N$ satisfying $\mu_{i}^{*}=\eta_{i}$ and $\omega \theta_{i}-\sum_{j \in N} a_{i j}^{*} m_{i j}\left(\mu^{*}\right)<\omega \eta_{i}$. From (8) this implies $\zeta_{i}\left(\mu^{*}\right)>0$ with $\mu_{i}^{*}=\eta_{i}$. Because $\mu^{*} \in M$ and, thus, $\mu^{*} \in \tilde{M}$, this contradicts the definition of $\eta_{i}$.

\section{The Bargaining Correspondence: Examples}

Working with Assumption 2, rather than with an explicitly specified model of the bargaining problem, makes our analysis applicable to a variety of bargaining models. We illustrate this with two examples giving rise to bargaining correspondences satisfying Assumption 2. The first example is based on Shimer and Smith (2000). The second example is based on Smith (2006) and establishes existence for the model in Lauermann and Nöldeke (2014).

Example 1 (Transferable Utility). The set of feasible flow payoffs in a relationship between players $i$ and $j$ is

$$
S_{i j}=\left\{\left(s_{i}, s_{j}\right) \in \mathbb{R}^{2} \mid s_{i}+s_{j} \leq s_{i j}\right\},
$$


where $s_{i j}=s_{j i} \in \mathbb{R}$ holds for all $(i, j) \in N \times N$. The bargaining protocol is as follows. In each meeting the two players are informed about each other's type. One player is randomly chosen to make a take-it-or-leave-it offer specifying a pair of flow payoffs $\left(s_{i}, s_{j}\right) \in S_{i j}$. The other player then either accepts or rejects that offer. If player $i$ is chosen as a proposer with probability $p_{i j} \in[0,1]$ and we consider the subgame perfect equilibria of the bargaining game induced by continuation values $v$, we have $(a, g) \in E(v)$ if and only if

$$
g_{i j}=p_{i j} \frac{\max \left\{s_{i j}-r v_{i}-r v_{j}, 0\right\}}{r+\omega}
$$

and

$$
a_{i j}= \begin{cases}0 & \text { if } s_{i j}<r v_{i}+r v_{j} \\ 1 & \text { if } s_{i j}>r v_{i}+r v_{j}\end{cases}
$$

for all $(i, j) \in N \times N$. This bargaining correspondence satisfies Assumption 2 with $\bar{g}=\max _{(i, j) \in N \times N} s_{i j} /(r+\omega)$.

Example 2 (Nontransferable Utility). The set of feasible flow payoffs contains a single point $\left(s_{i j}, s_{j i}\right) \in \mathbb{R}^{2}$ for every $(i, j) \in N \times N$. The bargaining protocol is that both players, being informed about each other's type, simultaneously declare whether they accept or refuse to match. The match forms if and only if both players accept. Eliminating dominated strategies, the equilibrium condition on the fraction $b_{i j} \in[0,1]$ of player $i$ who accept a player $j$ is

$$
b_{i j}= \begin{cases}0 & \text { if } s_{i j}<r v_{i} \\ 1 & \text { if } s_{i j}>r v_{i}\end{cases}
$$

The resulting matching probability is

$$
a_{i j}=b_{i j} b_{j i}
$$

and the corresponding gain is

$$
g_{i j}=b_{i j} b_{j i} \frac{s_{i j}-v_{i}}{r+\omega} .
$$

Therefore, $(a, u) \in E(v)$ if and only if there exists some $b \in[0,1]^{n \times n}$ satisfying (18) such that (19)-(20) hold. Assumption 2 holds with $\bar{g}=$ $\max _{(i, j) \in N \times N} s_{i j} /(r+\omega)$. 


\section{Discussion}

We have dispensed with uniqueness requirements used in previous proofs of the existence of steady-state equilibria in matching models with search frictions, thus enlarging the range of models for which existence can be shown. Some further extensions are easy to come by. For instance, our analysis carries over to a discrete - rather than continuous - time model without substantive changes. While we have considered infinitely lived agents who return to search whenever their current match is dissolved, our results are also applicable to an equivalent model with a stationary inflow of finitely lived agents (Eeckhout, 1999). Other extensions, e.g., allowing for a continuum of types or search by matched agents, require more work.

\section{References}

AdACHI, H. (2003): "A Search Model of Two-Sided Matching under Nontransferable Utility," Journal of Economic Theory, 113(2), 182-198.

Eeckhout, J. (1999): "Bilateral Search and Vertical Heterogeneity," International Economic Review, 40(4), 869-887.

Lauermann, S., And G. NÖldeke (2014): "Stable Marriages and Search Frictions," Journal of Economic Theory, 151, 163-195.

ManeA, M. (2014a): "Steady States in Matching and Bargaining," Mimeo, Department of Economics, MIT.

_ (2014b): "Bargaining in Dynamic Markets," Mimeo, Department of Economics, MIT.

Nöldeke, G., And T. TrÖGER (2009): "Matching Heterogeneous Agents with a Linear Search Technology," Bonn Econ Discussion Paper 1-2009, Bonn Graduate School of Economics.

Shimer, R., And L. Smith (2000): “Assortative Matching and Search," Econometrica, 68(2), 343-369.

Smith, L. (2006): "The Marriage Model with Search Frictions," Journal of Political Economy, 114(6), 1124-1144. 


\section{3, 319-338.}

Stevens, M. (2007): "New Microfoundations for the Aggregate Matching Function," International Economic Review, 48(3), 847-868. 